\title{
DEPRESSÃO PÓS-PARTO: IDENTIFICAÇÃO DE SINAIS, SINTOMAS E FATORES ASSOCIADOS EM MATERNIDADE DE REFERÊNCIA EM MANAUS
}

Sarah Regina Aloise', Alaidistania Aparecida Ferreira, Raquel Faria da Silva Lima

Objetivos: Identificar sinais e sintomas de Depressão Pós-Parto (DPP) e fatores associados em mulheres no puerpério mediato, entre 48h e 72h.Metodologia: estudo descritivo transversal com abordagem quantitativa realizado em maternidade de referência de Manaus- AM de junho a setembro de 2018. Foram aplicadas a versão brasileira da escala Edinburgh Postnatal Depression Scale- EPDS e formulário socioeconômico e obstétrico. Resultados: 15,06\% das 166 participantes apresentaram sinais e sintomas de DPP, porém não houve associação entre possível DPP e fatores socioeconômicos e clínico-obstétricos. Conclusões: o percentual de puérperas com score sugestivo de DPP encontra-se na média de outras pesquisas nacionais e a pesquisa mostrou ser eminente a identificação precoce de sinais e sintomas de DPP ainda no ambiente hospitalar 48h a 72h após o parto.

Descritores: Depressão Pós-Parto; Período Pós-Parto; Sinais e Sintomas.

\section{POSTPARTUM DEPRESSION: IDENTIFICATION OF SIGNS AND SYMPTOMS AND ASSOCIATED FACTORS IN REFERENCE MATERNITY HOSPITAL IN THE CITY OF MANAUS}

Objectives: To identify signs and symptoms of Postpartum Depression (PPD) and associated factors in women in postpartum between 48h and 72h.Methods: cross-sectional descriptive study with a quantitative approach performed in a reference maternity hospital of Manaus-AM from June to September 2018. The instruments used were the Brazilian version of the Edinburgh Postnatal Depression Scale-EPDS and socioeconomic and obstetric form.Results: $15.06 \%$ of 166 participants presented signs and symptoms of PPD, but there wasn't association between PPD and socioeconomic and clinical-obstetric factors.Conclusions: the percentage of postpartum women with a suggestive score of PPD is in the mean of other national surveys, and the research showed that is important the early identification of signs and symptoms of PPD at hospital in 48h-72h postpartum.

Descriptors: Depression Postpartum; Postpartum Period; Signs and Symptoms.

\section{DEPRESIÓN POST-PARTO: IDENTIFICACIÓN DE SIGNOS, SÍNTOMAS Y FACTORES ASOCIADOS EN LA MATERNIDAD DE REFERENCIA EN MANAUS.}

Objetivos: identificar signos y sintomas de Depresión Post-parto (DPP) y factores asociados en mujeres en puerpério mediato, entre 48h y 72h.Metodología: estudio descriptivo transversal con abordaje quantitativo realizado en maternidad de referencia de Manaus-AM de junio a septiembre de 2018. Los instrumentos utilizados fueron la versión brasileña de la escala Edinburgh Postnatal Depression Scale- EPDS y formulario socioeconómico y obstetrico.

Resultados: 15,06\% de 166 de las participantes presentaban signos y síntomas de DPP, pero no hicieron asociación entre posible DPP y factores socioeconómicos y clínicos-obstétricos.

Conclusión: El porcentaje de puérperas con puntuación sugestivo de DPP se encuentra en la media de otras pesquisas nacionales y la pesquisa mostró ser importante la identificación precoz de signos y síntomas de DPP en el ambiente hospitalario $48 \mathrm{~h}$ a $72 \mathrm{~h}$ pasado el parto.

Descriptores: Depresión Posparto; Período Posparto; Signos y Síntomas. 


\section{INTRODUÇÃO}

A gravidez é um período de grandes transformações no corpo feminino. Traz mudanças fisiológicas, sócio culturais e essencialmente emocionais, sendo os primeiros dias pósparto marcados por diversas emoções ${ }^{(1)}$. Nesse período de pós-parto ocorrem alterações de caráter emocional, observando-se maior vulnerabilidade da mãe e do bebê- o risco de a puérpera adquirir transtorno psiquiátrico mental é maior $^{(2)}$

Em se tratando de DPP, Barbosa ${ }^{(3)}$ afirma que a prevalência da doença no mundo é de 5 a 20\%. Particularmente no Brasil, os valores são de 12 a $37 \%{ }^{(4)}$ e estima-se a probabilidade de casos de DPP em pelo menos uma a cada quatro mulheres brasileiras ${ }^{(5)}$.

Tais dados são alarmantes, sendo motivo de preocupação no serviço de saúde. Trata-se de uma doença cujas consequências não se restringem a um único indivíduo: o núcleo familiar e principalmente mãe e bebê são atingidos. O relacionamento entre esse binômio é prejudicado e, segundo inúmeros estudos, isso pode afetar negativamente no desenvolvimento infantil. Conforme estudo realizado no Canadá, a relação entre a trajetória de DPP no período perinatal e a hiperatividade, desatenção, agressividade e ansiedade dos filhos mostrou-se significante(b)

Esse tipo de depressão ocorre durante um período em que o bebê é totalmente dependente de cuidados. Esse fato, somado à vulnerabilidade do estado emocional da puérpera, declara a urgência de cuidado multiprofissional ao binômio mãe-filho.

Constata-se, pois, a importância da identificação precoce de sinais e sintomas de DPP, bem como de fatores relacionados, a fim de prevenir agravos à saúde da mãe e atuar em conjunto com o núcleo familiar no processo saúdedoença, e dessa forma, estabelecer ações em prol do bemestar da família.

O profissional de enfermagem possui papel fundamental nesse processo, devendo ser capaz de identificar precocemente sinais e sintomas da doença e desenvolver ações em prol da saúde em nível individual e coletivo. (7)

Schardosim(8) evidencia o quão significativo são as escalas de rastreamento para identificar puérperas em risco de desenvolver DPP. Desenvolvida por Cox et al.(9), a escala Edinburgh Postnatal Depression Scale (EPDS) foi criada para identificar DPP, podendo ser utilizada em contexto clínico ou investigativo.

É um instrumento autoavaliativo que contém 10 questões baseadas em escalas previamente avaliadasIrritability, Depression, and Anxiety Scale; Hospital Anxiety and DepressionScale e Anxiety and Depression Scale ${ }^{(10)}$. As respostas são cotadas de zero a 3 , sendo cada item somado a fim de obter a pontuação total. Esta, se igual a 12 ou mais, indica probabilidade de depressão. É um instrumento simples, sendo a facilidade e rapidez de sua aplicação evidenciadas no ato da coleta de dados, momento este que não excede 10 minutos ${ }^{(11)}$. Além disso, é relevante ferramenta para detecção precoce de DPP(11), sendo um agente norteador do processo de planejamento da assistência à puérpera, bebê e família.

Diante disso, o objetivo desse estudo é identificar sinais e sintomas de DPP e determinar o perfil socioeconômico e obstétrico das participantes a fim de apontar possiveis fatores associados à doença.

\section{METODOLOGIA}

\section{Tipo de estudo}

Trata-se de um estudo descritivo transversal com abordagem quantitativa.

\section{Participantes da pesquisa}

Os sujeitos da pesquisa foram puérperas em período pósparto entre 48 e 72 horas, com 18 anos de idade ou mais e que falassem e compreendessem português, além de serem mães de recém-nascidos saudáveis. $O$ total da amostra foi de 166 mulheres.

\section{Local de estudo}

O estudo foi desenvolvido em uma maternidade pública de referência da cidade de Manaus.

\section{Coleta de dados}

A coleta foi realizada no período de junho a setembro de 2018, sendo aplicada a versão brasileira validada do instrumento (EPDS) por Santos et al(10). Utilizou-se também um formulário socioeconômico e obstétrico, criado pelas pesquisadoras, composto por 13 perguntas, relacionadas à idade, estado civil, número de filhos, número de gestações, tempo de internação na maternidade e de pós-parto, semana gestacional até o dia do parto, complicações no parto, gravidez atual desejada, escolaridade, renda familiar mensal, relacionamento familiar conflituoso, história de abuso sexual e caso de depressão anterior ao nascimento do bebê.

\section{Procedimentos de análise dos dados}

Para tabulação de dados utilizou-se o programa Microsoft Excel e as variáveis foram analisadas por meio do programa estatístico IBM SPSS Statistics versão 21 . Os resultados foram apresentados em tabelas e figuras (gráficos). Foi realizada análise exploratória e descritiva de todas as variáveis. As relações entre as variáveis sociais, clinico-obstétricas e psicológica e a presença de DPP foram verificadas por meio do teste não-paramétrico Qui-Quadrado de Pearson 
e as correlações entre essas mesmas variáveis, por meio do Coeficiente de Correlação de Spearman. Foi adotado um nível de $5 \%$ de significância para concluir as comparações.

Procedimentos éticos

A pesquisa foi aprovada pelo Comitê de ética em PesquisaCEP-em seres humanos da Universidade Federal do Amazonas (UFAM) sob número (CAAE: 84430218.9.0000.5020) e atendeu aos princípios éticos conforme Resolução n으 466/2012. (12)

As entrevistas ocorreram em ambiente reservado, garantindo a privacidade das entrevistadas e suas respostas. Antes do início de cada entrevista, foi apresentado e lido para cada puérpera o Termo de Consentimento Livre e Esclarecido (TCLE), assinado em duas vias pelas participantes. Foi assegurado o anonimato das entrevistadas e sigilo das informações, utilizadas apenas para fins científicos.

\section{RESULTADOS}

Foram avaliadas 166 mulheres, sendo que a idade delas variou entre 18 (mínima) e 43 (máxima) anos, com média correspondente de 27,25 $\pm 5,96$ anos de idade (Tabela 1). Com relação à faixa etária, 10 (6,02\%) mulheres tinham idade entre 18 e 20 anos (<20); 94 (56,63\%) na faixa etária de 20 a 29 anos e $62(37,35 \%)$ com 30 a 43 anos de idade.

Com relação ao estado civil dessas mulheres, 41 (24,70\%) são solteiras, 34 (20,48\%) casadas, 90 (54,22\%) mantêm uma união estável e 01 (0,60\%) declarou-se divorciada.

Quanto à escolaridade das mulheres que compuseram a amostra, 34 (20,28\%) possuem nível fundamental, 113 (68,07\%) nível médio, enquanto que apenas 19 (11,45\%) possuem nível superior.

No que se refere à renda econômica dessas mulheres, observou-se que $01(0,60 \%)$ não possui fonte de renda, 08 (4,92\%) recebem recurso financeiro referente ao programa social Bolsa Família (BF) e 27 (16, 27\%) possuem renda menor que um Salário Mínimo (SM). Dentre as que declararam receber a partir de um SM, 89 (53,61\%) possuem renda de 01 a 02 SM, 36 (21,69\%) com renda de 02 a 04 SM e 05 (3,01\%) de 04 a 05 SM. Atualmente, o valor do SM está fixo em 954 reais e BF até 177 reais por pessoa.

Quanto ao perfil clínico-obstétrico geral das puérperas entrevistadas, o número de filhos variou entre 01 (mínimo) e 10 (máximo), com valor mediano de $\mathrm{O} 2$ filhos por mulher (tabela 1). Já o número de gestações variou entre 01 (mínima) e 10 (máxima), com valor mediano de 02 gestações por mulher. $\mathrm{O}$ tempo mediano de internação foi de 03 dias, variando entre 02 (tempo mínimo) e 26 (tempo máximo) dias de internação (tabela 1).

Em relação semana gestacional até o dia do parto, o número mínimo de semanas encontrado foi de 35 e o máximo de 42, com número médio de 39,19 \pm 1,34 semanas (tabela 1).

Tabela 1 - Análise Descritiva das Variáveis Quantitativas

\begin{tabular}{|c|c|c|c|c|c|c|}
\hline \multirow{2}{*}{ VARIÁVEL } & \multirow{2}{*}{$\begin{array}{c}\mathbf{n} \\
\text { pacientes }\end{array}$} & \multicolumn{5}{|c|}{ MEDIDAS DESCRITIVAS } \\
\hline & & Média & dp & Mínimo & Mediana & Máximo \\
\hline Idade & 166 & 27,25 & 5,97 & 18 & 26 & 43 \\
\hline $\mathrm{N}^{\circ}$ de filhos & 166 & 2,27 & 1,41 & 1 & 2 & 10 \\
\hline $\mathrm{N}^{\circ}$ de gestações & 166 & 2,58 & 1,59 & 1 & 2 & 10 \\
\hline Tempo de & & & & & & \\
\hline internação (dias) & 166 & 2,99 & 2,14 & 2 & 3 & 26 \\
\hline Semana gestacional & 166 & 39,19 & 1,34 & 35 & 39 & 42 \\
\hline
\end{tabular}

A Tabela 2 abaixo mostra as características clínicoobstétricas das puérperas entrevistas, referentes ao número de filhos, número de gestações, período pós-parto, tempo de internação, semana gestacional até o dia do parto, via de parto, complicações durante o parto e gravidez desejada.

Tabela 2 - Características clínico-obstétricas das pacientes avaliadas

\begin{tabular}{|c|c|c|}
\hline CARACTERÍSTICAS & $\mathbf{n}^{*}$ & $\%$ \\
\hline \multicolumn{3}{|l|}{$\mathrm{N}^{\circ}$ de Filhos } \\
\hline 01 a 03 & 143 & 86,14 \\
\hline 04 a 06 & 21 & 12,65 \\
\hline$>06$ & 2 & 1,20 \\
\hline \multicolumn{3}{|l|}{$\mathbf{N}^{\circ}$ de Gestações } \\
\hline 01 a 03 & 134 & 80,72 \\
\hline 04 a 06 & 27 & 16,27 \\
\hline 07 a 10 & 5 & 3,01 \\
\hline \multicolumn{3}{|l|}{ Pós-Parto } \\
\hline $48 \mathrm{~h}$ & 135 & 81,33 \\
\hline $72 \mathrm{~h}$ & 31 & 18,67 \\
\hline \multicolumn{3}{|l|}{ Tempo de internação (dias) } \\
\hline Até 03 & 148 & 89,16 \\
\hline 04 a 06 & 15 & 9,04 \\
\hline$>06$ & 3 & 1,81 \\
\hline \multicolumn{3}{|c|}{ Semana gestacional até o parto } \\
\hline 35 a 37 & 21 & 12,65 \\
\hline 38 a 40 & 124 & 74,70 \\
\hline$>40$ & 21 & 12,65 \\
\hline \multicolumn{3}{|l|}{ Via do Parto } \\
\hline Cesáreo & 109 & 65,66 \\
\hline Vaginal & 57 & 34,34 \\
\hline \multicolumn{3}{|l|}{ Complicações no parto } \\
\hline $\operatorname{Sim}$ & 32 & 19,28 \\
\hline Não & 134 & 80,72 \\
\hline \multicolumn{3}{|l|}{ Gravidez Desejada } \\
\hline $\operatorname{Sim}$ & 90 & 54,22 \\
\hline Não & 76 & 45,78 \\
\hline
\end{tabular}


Vale destacar que a coleta de dados foi mais representativa em mulheres de Parto Cesáreo (PC) devido ao tempo de permanência maior destas na unidade, somado a maior probabilidade desse grupo atender aos critérios de inclusão de tempo de pós-parto- $48 \mathrm{~h}$ e $72 \mathrm{~h}$. Portanto, os resultados das variáveis relacionados a PC e Partos Vaginais (PV) foram obtidos devido aos critérios de inclusão da pesquisa e não declaram a quantidade de PC e PV ocorridos na maternidade no período da coleta.

Nas 32 mulheres que tiveram complicações durante o parto, houve 34 ocorrências ao todo, sendo que a mais frequente foi a Hipertensão Arterial Sistêmica (HAS), que ocorreu em 18 mulheres e representou 52,94\% de todas as complicações ocorridas. Neste sentido, as 03 complicações mais frequentes, dentre as 34 foram: HAS (18; 52,94\%), Ausência de dilatação (03; 8,82\%) e Hipotensão (03; 8,82\%). Hipóxia do recém-nascido (2; 5,88\%); Dispneia (2; 5,88\%); Pré-eclâmpsia (1; 2,94\%); Presença de mecônio (1; 2,94\%); Hemorragia (1; 2,94\%); Diabetes Mellitus Gestacional (1; 2,94\%); Cirurgia de retirada de cisto (1; 2,94\%) e Batimento Cardíaco Fetal >160bpm (1; 2,94\%) também foi relatado pelas participantes.

$\mathrm{Na}$ avaliação dessas mulheres, foi observado que, das 166, 15 (9,04\%), tiveram conflitos familiares, 07 (4,22\%) possuem histórico de abuso sexual e 18 (10,84\%) tiveram depressão anterior ao nascimento do bebê.

O resultado da EPDS apontou que das 166 mulheres, 25 (15,06\%) tinham probabilidade de estarem com DPP, enquanto que 141 (84,94\%) não apresentaram pontuação suficiente que garantisse resultado positivo para sinais e sintomas de DPP (figura l)

Figura 1 - Proporção de mulheres com sinais e sintomas de DDP

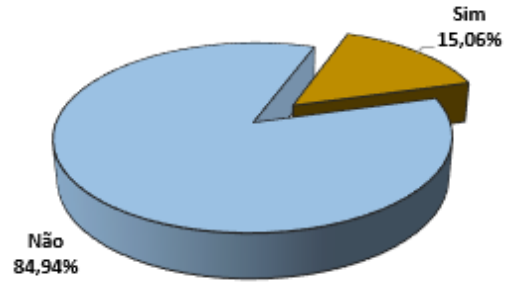

Ao verificar se existia alguma relação entre as variáveis sociais, econômicas e clinico-obstétricas com a presença de DDP, observou-se que não houve evidências de que exista relação estatisticamente significativa entre a ocorrência desta e as referidas variáveis, se considerarmos uma significância de 5\%. Entretanto, é importante enfatizar que considerando uma significância de 10\%, as variáveis faixa etária, escolaridade e abuso tendem a estar relacionadas à ocorrência de DPP (tabela 3).

Tabela 3 - Relação entre os fatores sociais, econômicos, clínicos e obstétricos com a presença de DPP

\begin{tabular}{|c|c|c|c|c|c|c|}
\hline \multirow{2}{*}{ FATORES } & \multicolumn{4}{|c|}{ DEPRESSÃO PÓS-PARTO } & \multirow{2}{*}{$\mathbf{n}$} & \multirow{2}{*}{$p^{*}$} \\
\hline & Sim & $\%$ & Não & $\%$ & & \\
\hline \multicolumn{7}{|l|}{ Faixa Etária } \\
\hline$<20$ & 4 & 40,00 & 6 & 60,00 & 10 & \multirow{4}{*}{0,061} \\
\hline 20 a 29 & 14 & 14,89 & 80 & 85,11 & 94 & \\
\hline 30 a 43 & 7 & 11,29 & 55 & 88,71 & 62 & \\
\hline Total & 25 & 15,06 & 141 & 84,94 & 166 & \\
\hline \multicolumn{7}{|l|}{ Estado Civil } \\
\hline Solteira & 10 & 24,39 & 31 & 75,61 & 41 & \multirow{5}{*}{0,269} \\
\hline Casada & 3 & 8,82 & 31 & 91,18 & 34 & \\
\hline União Estável & 12 & 13,33 & 78 & 86,67 & 90 & \\
\hline Divorciada & 0 & 0,00 & 1 & 0,61 & 165 & \\
\hline Total & 25 & 7,58 & 141 & 42,73 & 330 & \\
\hline \multicolumn{7}{|l|}{$N^{\circ}$ de Filhos } \\
\hline 01 a 03 & 20 & 13,99 & 123 & 86,01 & 143 & \multirow{4}{*}{0,360} \\
\hline 04 a 06 & 4 & 19,05 & 17 & 80,95 & 21 & \\
\hline$>06$ & 1 & 50,00 & 1 & 50,00 & 2 & \\
\hline Total & 25 & 15,06 & 141 & 84,94 & 166 & \\
\hline \multicolumn{7}{|l|}{$\mathbf{N}^{\circ}$ de Gestações } \\
\hline 01 a 03 & 17 & 12,69 & 117 & 87,31 & 134 & \multirow{4}{*}{0,191} \\
\hline 04 a 06 & 7 & 25,93 & 20 & 74,07 & 27 & \\
\hline 07 a 10 & 1 & 20,00 & 4 & 80,00 & 5 & \\
\hline Total & 25 & 15,06 & 141 & 84,94 & 166 & \\
\hline \multicolumn{7}{|l|}{$\begin{array}{l}\text { Tempo de internação } \\
\text { (dias) }\end{array}$} \\
\hline Até 03 & 22 & 14,86 & 126 & 85,14 & 148 & \multirow{4}{*}{0,820} \\
\hline 04 a 06 & 2 & 13,33 & 13 & 86,67 & 15 & \\
\hline$>06$ & 1 & 33,33 & 2 & 66,67 & 3 & \\
\hline Total & 25 & 15,06 & 141 & 84,94 & 166 & \\
\hline \multicolumn{7}{|l|}{ Semanas de gestação } \\
\hline 35 a 37 & 3 & 14,29 & 18 & 85,71 & 21 & \\
\hline 38 a 40 & 21 & 16,94 & 103 & 83,06 & 124 & 0,191 \\
\hline$>40$ & 1 & 4,76 & 20 & 95,24 & 21 & \\
\hline Total & 25 & 15,06 & 141 & 84,94 & 166 & \\
\hline Via de Parto & & & & & & \\
\hline Parto Cesárea & 19 & 17,43 & 90 & 82,57 & 109 & \\
\hline Parto Vaginal & 6 & 10,53 & 51 & 89,47 & 57 & 0,238 \\
\hline Total & 25 & 15,06 & 141 & 84,94 & 166 & \\
\hline Complicações & & & & & & \\
\hline Sim & 8 & 25,00 & 24 & 75,00 & 32 & \\
\hline Não & 17 & 12,69 & 117 & 87,31 & 134 & 0,284 \\
\hline Total & 25 & 15,06 & 141 & 84,94 & 166 & \\
\hline Gravidez desejada & & & & & & \\
\hline Sim & 11 & 12,22 & 79 & 87,78 & 90 & \\
\hline Não & 14 & 18,42 & 62 & 81,58 & 76 & 0,266 \\
\hline Total & 25 & 15,06 & 141 & 84,94 & 166 & \\
\hline Escolaridade & & & & & & \\
\hline Ensino Fundamental & 9 & 26,47 & 25 & 73,53 & 34 & \\
\hline Ensino Médio & 15 & 13,27 & 98 & 86,73 & 113 & 0.073 \\
\hline Ensino Superior & 1 & 5,26 & 18 & 94,74 & 19 & 0,073 \\
\hline Total & 25 & 15,06 & 141 & 84,94 & 166 & \\
\hline Conflito familiar & & & & & & \\
\hline $\operatorname{Sim}$ & 4 & 26,67 & 11 & 73,33 & 15 & \\
\hline Não & 21 & 13,91 & 130 & 86,09 & 151 & 0,247 \\
\hline Total & 25 & 15,06 & 141 & 84,94 & 166 & \\
\hline Abuso sexual & & & & & & \\
\hline $\operatorname{Sim}$ & 3 & 42,86 & 4 & 57,14 & 7 & \\
\hline Não & 22 & 13,84 & 137 & 86,16 & 159 & 0,070 \\
\hline Total & 25 & 15,06 & 141 & 84,94 & 166 & \\
\hline Depressão anterior & & & & & & \\
\hline Sim & 5 & 27,78 & 13 & 72,22 & 18 & \\
\hline Não & 20 & 13,51 & 128 & 86,49 & 148 & 0,154 \\
\hline Total & 25 & 15,06 & 141 & 84,94 & 166 & \\
\hline
\end{tabular}

Enferm. Foco 2019: 10 (3): 41-45 | 43 
*Valores de p são significativos para p < 0,05 (5\%)

Correlação de Spearman

DISCUSSÃO

Das 166 participantes, 25 (15,06\%) apresentaram pontuação sugestiva de DPP. O valor em porcentagem se aproxima de resultados encontrados em demais pesquisas como a de Hartmann ${ }^{(13)}$ com 14\% e Araújo ${ }^{(14)}$ com 19,8\%, apesar de outras apresentarem valores ainda mais altos- Theme Filha $^{(5)}$ de 26,3\% e Figueira ${ }^{(15)}$ com 26,9\%. Todavia, não houve significativa relação estatística entre as variáveis e possivel DPP. Consequentemente não foi possivel relacionar fatores de risco associados à DPP, embora tenha-se observado matematicamente que, quanto maior o nivel de escolaridade, maior é a chance de ocorrer DPP, igualmente quando há histórico de abuso sexual. Pesquisadoras da Austrália apontam elevadas taxas de depressão em mulheres que já sofreram abuso sexual ${ }^{(16)}$.

Em estudo realizado em Belo Horizonte, por Figueira ${ }^{(15)}$ igualmente não houve associação entre idade, escolaridade, número de partos anteriores e estado civil com o risco de DPP. Já em outros trabalhos, houve associação de fatores de risco, como paridade maior ou igual a dois ${ }^{(5,13)}$, baixa classe econômica e gravidez não planejada ${ }^{(5)}$ e baixa escolaridade ${ }^{(17)}$. A partir disso, observam-se diferentes resultados de pesquisas que utilizam a EPDS para investigação de DPP, e relacionados a essa questão, pode-se citar a adoção de diferentes escores de pontuação para possivel DPP. Essa pesquisa utilizou escore maior ou igual a 12, conforme artigo original da EPDS ${ }^{(9)}$ e Morais ${ }^{(18)}$. Porém, outros estudos adotaram escore a partir de $10^{(13,15,17)}$, maior ou igual a $11^{(11)}$ e maior ou igual a $13^{(5)}$, não havendo, portanto, um consenso quanto a pontuação mínima efetiva de sinais e sintomas de DPP.

Outro ponto a considerar é o estado psíquico e emocional da puérpera no momento da entrevista. Pode haver constrangimento ou negação de determinadas situações, e consequentemente respostas que não retratem a realidade da mãe. Todavia, conforme observadas pontuações iguais ou acima de 12, o setor de psicologia da maternidade era acionado. Assim, o acompanhamento das puérperas pelo serviço especializado assistiu e constatou sintomas prodrômicos.

\section{Limitações do estudo}

Pode-se apontar como limitação do estudo o seguimento transversal da pesquisa, visto que a aplicação da escala de EPDS em outros momentos poderia avaliar a persistência de sinais e sintomas da doença.

\section{Contribuição do estudo para a prática}

O estudo ressalta a importância do diagnóstico precoce de DPP nas instituições de saúde, bem como a possibilidade de se identificar sinais e sintomas através da aplicação do EPDS. O papel da equipe multiprofissional e em especial da enfermagem nesse processo é primordial.

\section{CONCLUSÃO}

Diante disso, foi possível identificar sinais e sintomas de DPP em 25 das 166 mulheres em puerpério mediato, entre 48h e 72h. Todavia, ao relacionar as variáveis sociais, econômicas e clinico-obstétricas com a presença de DDP, observou-se não haver significância de fatores associados à DPP.

A aplicação da EPDS serve de ferramenta de auxílio no trabalho multiprofissional em saúde- particularmente na Enfermagem como apoio à assistência nos níveis primário (19) e terciário(11) com embasamento científico. É importante ressaltar que escalas de rastreamento de DPP não indicam diagnóstico, e sim probabilidade da doença, sendo o uso da escala de grande relevância para a detecção precoce de sinais e sintomas de DPP(10).

\section{Contribuição dos autores}

Concepção e/ou desenho: Aloise SR, Ferreira AA, Lima RFS; Análise e interpretação dos dados, redação do artigo: Aloise SR; Revisão crítica e Revisão final: Aloise SR, Ferreira AA, Lima RFS. 


\section{REFERÊNCIAS}

1- Pereira FM. Sintomas depressivos no puerpério: uma revisão de literatura [Internet]. São Paulo: Marilia; 201l. Disponivel em: http://pesquisa.bvsalud.org/bvsvs/ resource/pt/ses-31110

2- Valença CN, Germano RM. Prevenindo a depressão puerperal na estratégia saúde da familia: Ações do enfermeiro no pré-natal. Rev Rene. Fortaleza. 2010; 11(2):129-39. Disponivel em: http://periodicos.ufc.br/rene/article/view/4541. Acesso em 13 de agosto de 2019.

3- Barbosa RCA. Prevalência e Incidência de Depressão Pós-Parto e Sua As3- Barbosa RCA. Prevalencia e Incidencia de Depressão Pós-Parto e Sua As-
sociaço com o Apoio Social [dissertação]. Recife, PE: Universidade Fedesociação com o Apoio Social [dissertação]. Recife, PE: Universidade Fede-
ral de Pernambuco: 2008. Disponivel em: https://repositorio.ufpe.br/bitstream/123456789/9231/1/arquivo4156_l.pdf

4- Moraes GPA, Lorenzo L, Pontes GAR, Montenegro MC, Cantilino A. Screening and diagnosing postpartum depression: when and how? Trends Psychiatry Psychother. 2017:39(1) - 54-61. Disponivel em: http://www.scielo.br/scielo.php?script=sci_arttext\&pid=\$2237-60892017000100054

5- Theme Filha MM, Ayers S, Gama SGN, Leal MC. Factors associated with postpartum depressive symptomatology in Brazil: The Birth in Brazil National Research Study, 2011/2012. J Affect Disord. 2016; 194:159-67. Disponivel em: https:// arch Study, 2011/2012. J Affect Disord. 2016; 194:159-67. Disponivel em: https:// Acesso em 30 de maio de 2019.

6- Kingston D, Kehler H, Austin MP, Mughal MK, Wajid A, Vermeyden L, et al. Trajectories of maternal depressive symptoms during pregnancy and the first 12 months postpartum and child externalizing and internalizing behavior at three years. PLoS ONE. 2018 Apr 13: (4). Disponivel em: https://journals.plos.org/plosone/article?id=10.1371/journal.pone.0195365

7- Brasil. Conselho Nacional de Educação. Câmara de Educação Superior. Resolução no 3/2001. Diário Oficial da União, Brasilia. 2001 Nov 9: Sect 1: 37. Disponivel em: <http://portal.mec.gov.br/cne/arquivos/pdf/CESO3.pdf> Acesso em 3 de dezembro de 2018 .

8- Schardosim J M. Heldt E. Escalas de rastreamento para depressão pós-parto: uma revisão sistemática. Rev Gaúcha Enferm. (Online). Porto Alegre. 2011 Mar: 32(1):159-66. Disponivel em: <http://www.scielo.br/scielo.php?script=sci_arttextEpid=S1983-14472011000100021Elng=enEnrm=iso >

9- Cox JL, Holden JM, Sagovsky R. Detection of postnatal depression: development of the 10-item Edinburgh Postnatal Depression Scale. Br J Psychiatry. 1987 150:782-86. Disponivel em: <https://www.cambridge.org/core/journals/the-bri150:782-86. Disponivel em: <https://www.cambridge.org/core/journals/the-bri-
tish-journal-of-psychiatry/article/detection-of-postnatal-depression/El8BCtish-journal-of-psychiatry/article/detection-of-postnatal-depressio
62858DBF2640C33DCC8B572F02A> Acesso em 17 de junho de 2019.

10- Santos IS, Matijasevich A, Tavares BF, Barros AJD, Botelho IP, Lapolli C, et al. Validation of the Edinburgh Postnatal Depression Scale (EPDS) in a sample of mothers from the 2004 Pelotas Birth Cohort Study. Cad Saúde Pública. Rio de Janeiro. 2007; 23(11):2577-88. Disponivel em:
12- Brasil. Ministério da Saúde. Conselho Nacional de Saúde. Resolução no 466/2012. Diário Oficial da Uniāo. Brasilia 2012 Dez 12. Disponivel em: <http://bvsms.saude.gov.br/bvs/saudelegis/cns/2013/res0466_12_12_2012.html> Acesso em 23 de junho de 2019 .

13- Hartmann JM, Sassi RAM, Cesar JA. Depressão entre puérperas: prevalência e fatores associados. Cad Saúde Pública. 2017; 33(9):e00094016. Disponivel em: <http://www.scielo.br/pdf/csp/v33n9/1678-4464-csp-33-09-e00094016.pdf> Acesso em 30 de maio de 2019.

14. Araújo IS, Aquino KS, Fagundes LKA, Santos VC. Postpartum Depression: Epidemiological Clinical Prole of Patients Attended In a Reference Public Maternity in Salvador-BA. Rev Bras Ginecol Obstet. 2019:41(3):155-63.

15- Figueira P, Corrêa H, Malloy-Diniz L. Romano- Silva MA. Escala de depressão pós-natal de Edimburgo para triagem no sistema público de saúde. Rev Saúde Pública. 2009: 43 Suppl 1:79-84. Disponivel em: <http://www.scielo.br/scielo.php?script $=$ sci_arttext8pid=S0034-89102009000800012>. Acesso em 18 de junho de 2019.

16- Buist A, Janson H. Childhood sexual abuse, parenting and postpartum depression: A 3-year follow-up study. Child Abuse Negl. 2001; 25, 909-21. Disponivel em: <https://www.sciencedirect.com/science/article/abs/pii/S0145213401002460?via\%3Dihub> Acesso em 18 de junho de 2019

17- Melo SB, Jordão RRR, Guimarães FJ, Perrelli JGA, Cantilino A, Sougey FB Sintomas depressivos em puérperas atendidas em Unidades de Saúde da Familia. Rev Bras Saúde Mater Infant. Recife. Jan-mar 2018; 18(1): 17177. Disponivel em: <http://www.scielo.br/scielo.php?script=sci_arttext\&pi$d=\$ 1519-38292018000100163$ \&lng=en\&nrm=iso\&tlng=pt> Acesso em 24 de junho de 2019

18- Morais MLS, Fonseca LAM, David VF, Viegas LM, Otta E. Fatores psicossociais e sociodemográficos associados à depressão pós-parto: Um estudo em hospitais público e privado da cidade de São Paulo. Brasil Estud Psicol (Natal). 2015 Janpúblico e privado da cidade de São Paulo, Brasil. Estud Psicol (Natal). 2015 Jan-
-Mar: 20(1): 40-9. Disponivel em: http://dx.doi.org/10.5935/1678-4669.20150006 Acesso em 3 de julho de 2019

19- Félix TA, Ferreira AGN, Siqueira DA, Nascimento KV, Ximenes Neto FRG, Mira QL. Atuação da enfermagem frente à depressão pós-parto nas consultas de puericulturas. Rev Enfermeria Global. 2013 Jan; (29): 420-35. Disponivel em: <http:// scielo.isciii.es/pdf/eg/v12n29/pt_enfermerial.pdf > Acesso em 12 de agosto de 2019 